\title{
Relative Humidity Profile Estimation Method with AIRS (Atmospheric Infrared Sounder) Data by Means of SDM (Steepest Descend Method) with the Initial Value Derived from Linear Estimation
}

\author{
Kohei Arai $^{1}$ \\ ${ }^{1}$ Graduate School of Science and Engineering \\ Saga University \\ Saga City, Japan
}

\begin{abstract}
Relative humidity profile estimation method with AIRS (Atmospheric Infrared Sounder) data by means of SDM (Steepest Descend Method) with the initial value derived from LED: Linear Estimation Method is also proposed. Through experiments, it is found that there is almost $15(\%)$ of relative humidity estimation error. Therefore, it can be said that the relative humidity is still tough issue for retrieval. It is also found that the estimation error does not depend on the designated atmospheric models, Mid-Latitude Summer/Winter, Tropic. Even if the assigned atmospheric model is not correct, the proposed SDM based method allows almost same estimated relative humidity. In other word, it is robust against atmospheric model.
\end{abstract}

Keywords-Atmospheric Infrared Sounder (AIRS); Steepest Descend Method (SDM); LED; MODTRAN; relative humidity; atmospheric model; Infrared sounder

\section{INTRODUCTION}

Vertical profiles of air-temperature and relative humidity (Water vapor) can be estimated with satellite based Infrared Sounder data [1]. Retrieval accuracy, however, is not good enough for Earth system science. Estimation accuracy of airtemperature and relative humidity at tropopause ${ }^{1}$ altitude is not good enough, in particular, because there are gradient changes of air-temperature and relative humidity profile in the tropopause. Therefore, observed radiance at the specific channels is not changed for the altitude.

There is least square based conventional method for estimation of vertical profiles of air-temperature and relative humidity. In the method, Root Mean Square (RMS) difference between observed radiance and calculated radiance (model based radiance) with the designated physical parameters in the model is minimized. Then, the designated physical parameters including air-temperature and relative humidity at the minimum RMS difference are to be solutions.

The most typical least square method is Newton-Raphson method $^{2}$ which gives one of local minima. Newton-Raphson method needs the first and the second order derivatives,

\footnotetext{
${ }^{1}$ http://en.wikipedia.org/wiki/Tropopause

2 http://en.wikipedia.org/wiki/Newton's_method
}

Jacobean and Hessian at around the current solution. It is not easy to formularize these derivatives analytically. The proposed method is based on Levenberg Marquardt (LM) ${ }^{3}$ of non-linear least square method. It uses numerically calculated the first and the second order derivatives instead of analytical based derivatives, namely, these derivatives can be calculated with radiative transfer model based radiance calculations. At around the current solution in the solution space, directional derivatives are calculated with the radiative transfer model such as MODTRAN.

The proposed method is validated for air-temperature and relative humidity profile retrievals with Infrared: IR sounder ${ }^{4}$ data obtained from AQUA/AIRS (AIRS instrument onboard AQUA satellite [2]-[7] by mean of Steepest Descent Method: SDM. Although SDM based optimization method shows relatively good accuracy, it takes a comparatively large computer resources and it is falling in local minima, not in the global optimum. To avoid this situation of which the solution is falling in local minima and accelerate the convergence through giving initial value which is derived from a Linear Estimation Method (LEM). Due to the fact that retrieval accuracy depends on the initial value, the most appropriate initial value is given from the LEM in the proposed method.

From the previous research works, a comparison of retrieving accuracy between Newton-Raphson method and the proposed method based on LM method [8] is made to demonstrate an effectiveness of the proposed method in terms of estimation accuracy for the altitude of tropopause [9]. Global Data Assimilation System: GDAS ${ }^{5}$ data of assimilation model derived 1-degree mesh data is used as truth data of airtemperature and relative humidity profiles. The experimental data show that the proposed method is superior to the conventional Newton-Raphson method.

The next section describes the proposed method together with typical conventional Newton-Raphson method for retrieving vertical profiles followed by experiments. Then

\footnotetext{
${ }^{3}$ http://en.wikipedia.org/wiki/Levenberg\%E2\%80\%93Marquardt_algorithm

${ }^{4}$ http://en.wikipedia.org/wiki/Atmospheric_Infrared_Sounder

${ }^{5} \mathrm{http} / / / \mathrm{www} . \mathrm{mmm}$.ucar.edu/mm5/mm5v3/data/gdas.html
} 
conclusion with some discussions is followed by together with future research works.

\section{PROPOSED METHOD}

\section{A. Infrared Sounder}

Fig. 1 shows atmospheric transmittance and transmittance of carbon dioxide and water vapor. Using absorption bands of molecules, carbon dioxide and water vapor content in the atmosphere can be retrieved. There is strong relation between air-temperature and carbon dioxide content. Meanwhile, water vapor content is a closely related to relative humidity. Therefore, air-temperature and relative humidity can be estimated using absorption characteristics of carbon dioxide and water vapor contents in the atmosphere.

Using slope characteristics of the absorption characteristics, vertical profile of carbon dioxide and water vapor can also be estimated. Optical depths ${ }^{6}$ of molecules are different and depend on the wavelength at the slope of the absorption characteristics. First derivative of optical depth against altitude is called weighting function. ${ }^{7}$ Therefore, weighting function is a function of altitude.

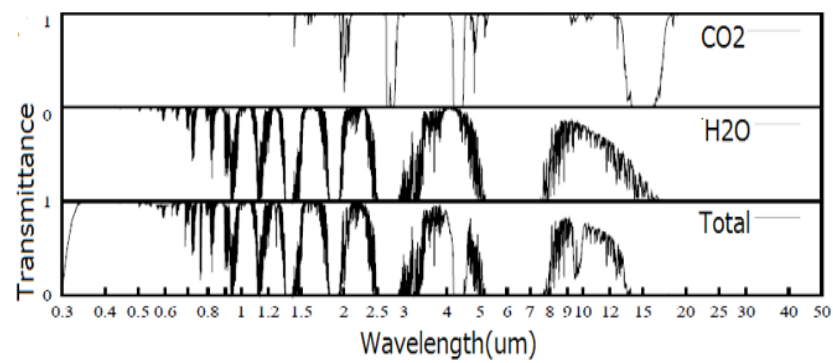

Fig. 1. Atmospheric transmittance and transmittance of carbon dioxide and relative humidity.

Fig. 2(a) shows an example of a transmittance as a function of altitude while (b) shows and example of a weighting function.

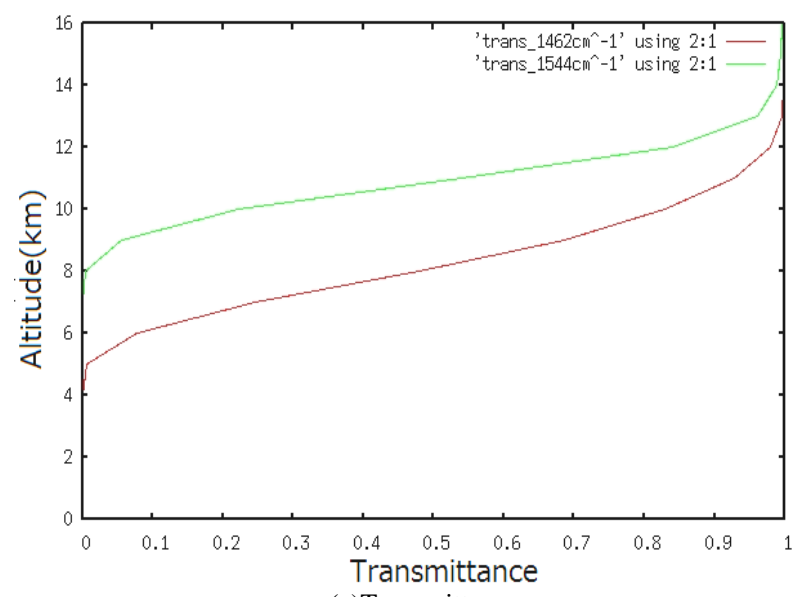

(a)Transmittance

\footnotetext{
${ }_{7}^{6} \mathrm{http} / / /$ en.wikipedia.org/wiki/Optical_depth

http://www.ecmwf.int/newsevents/training/rcourse_notes/DATA_ASSIMILA TION/INVERSION_METHODS/Inversion_methods2.html
}

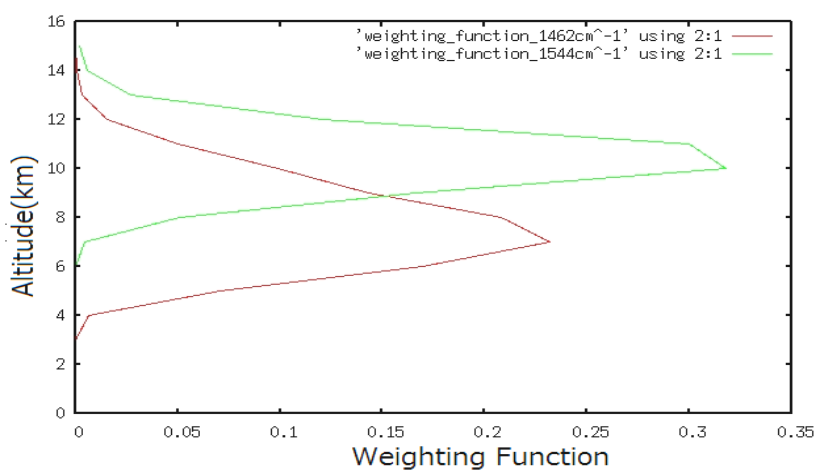

(b) Weighting function

Fig. 2. Transmittance as a function of altitude and weighting function.

Mission instruments which allow vertical profile retrievals in the infrared wavelength region onboard satellite are called as infrared sounder. There are some infrared sounders such as TIROS-N / NOAA TOVS- High Resolution Infrared Sounder model $2(\mathrm{HIRS} / 2)^{8}$. One of infrared sounders which are widely used with high quality of mission instruments is $\operatorname{AIRS}^{9}$ : Atmospheric Infrared Sounder. Although AIRS data quality is high enough, retrieval accuracy is not good enough. Estimation accuracy of air-temperature and relative humidity at around tropopause is not good enough.

\section{B. Conventional Retrieval Method}

The previously proposed method for air-temperature and relative humidity retrievals is intended to improve retrieval accuracy. Typically, the conventional retrieval method is minimizing formulated error covariance using NewtonRaphson method. It, however, gives one of local minima sometime. Although the simulated annealing method gives global optimum solution, it takes huge computational resources.

The proposed method is based on non-linear least square method of Levenberg Marquardt: LM. LM method minimizes the difference between AIRS data derived radiance, $R_{0 i}$ and estimated radiance, $R_{i}$ based on atmospheric model with the parameters of air-temperature and relative humidity.

$$
S=\sum_{i=0}^{n}\left(R_{i}-R_{0 i}\right)^{2}
$$

Thus, the geophysical parameter of air temperature and water vapor (relative humidity) at the difference of radiance reaches to the minimum is estimated. Widely used, reliable and accurate enough atmospheric software code of MODTRAN $^{10}$ which allows calculate at sensor radiance with a variety of parameters is used in the proposed method. Solution update equation of Newton-Raphson method is expressed by (2).

$$
\mathrm{X}_{\mathrm{n}+1}=\mathrm{X}_{\mathrm{n}}-\mathrm{H}^{-1} \mathrm{~J}\left(\mathrm{X}_{\mathrm{n}}\right)
$$

\footnotetext{
${ }^{8}$ www.ozonelayer.noaa.gov/action/tovs.htm

${ }^{9} \mathrm{http}: / /$ aqua.nasa.gov/about/instrument_airs.php

${ }^{10} \mathrm{http} / / / \mathrm{en}$.wikipedia.org/wiki/MODTRAN
} 
where $H$ denotes Hessian matrix ${ }^{11}$ which consists of the second order derivatives (residual square error, $S$ which is represented by (1) by geophysical parameter, air-temperature and water vapor (relative humidity)). Also, $J$ denotes Jacobean ${ }^{12}$ which consists of the first derivative of vectors $(S$ by geophysical parameters of air temperature and water vapor (relative humidity), $x$ ). On the other hand, solution update equation is expressed by (3).

$$
X_{n+1}=X_{n}+\left(J^{T} J+I\right)^{-1} J^{T}\left(R_{i}-R_{0 i}\right)
$$

The first derivative is represented by (4) while the second order derivative is expressed by (5), respectively.

$$
\begin{aligned}
& \frac{\partial S}{\partial x_{i}}=-2 \sum_{k=1}^{n}\left(R_{k-} R_{0 k}\right) \frac{\partial R_{0 k}}{\partial x_{i}} \\
& \frac{\partial^{2} S}{\partial x_{i} x_{j}}=2 \sum_{k=1}^{n}\left[\frac{\partial R_{0 k}}{\partial x_{i}}-\frac{\partial R_{0 k}}{\partial x_{j}}-\left(R_{k}-R_{0 k}\right) \frac{\partial^{2} R_{0 k}}{\partial x_{i} \partial x_{j}}\right]
\end{aligned}
$$

On the other hand, the first and second order derivatives of $R$ with $x$ are expressed by (6) and (7), respectively.

$$
\begin{aligned}
& \frac{\partial R_{0 \mathrm{k}}}{\partial x_{i}} \leftarrow \text { MODTRAN } \\
& \frac{\partial^{2} R_{0 \mathrm{k}}}{\partial x_{i} \partial x_{j}} \leftarrow \frac{\partial R_{0 \mathrm{k}}}{\partial x_{i}} * \frac{\partial R_{0 \mathrm{k}}}{\partial x_{j}}
\end{aligned}
$$

Equation (6) can be derived from MODTRAN. Thus, the geophysical parameter, air temperature and water vapor relative humidity at the difference of radiance reaches to the minimum is estimated.

In the previously proposed method, these derivatives are calculated numerically. In order to determine the derivatives, $2 \%$ changes of relative humidity are considered for calculation of derivative $R$ and $S$ while $0.5 \mathrm{~K}$ changes of air-temperature is also considered. Thus, the geophysical parameter of air temperature and water vapor (relative humidity) at when the difference of radiance reaches to the minimum is estimated.

\section{Proposed Retrieval Method}

Radiative transfer equation is expressed as follows:

$$
R v=\left(I_{0}\right)_{v} \tau_{v}\left(z_{0}\right)+\int_{z_{0}}^{\infty} B v\{T(z)\} K_{v}(z) d z
$$

where $R, I, \tau, z, B, T, K$ denotes the top of the atmosphere radiance (at sensor radiance), extinction component from the atmosphere, transparency of the atmosphere, altitude, brightness temperature of the ground surface, surface temperature, extinction function, respectively. This can be simplified and rewritten as follows:

$$
R=B K
$$

Then, $\mathrm{R}$ can be minimized as (10) through changing atmospheric transparency which is closely related to relative humidity content in the atmosphere which results in relative humidity profile retrieval.

\footnotetext{
${ }^{11} \mathrm{http}: / /$ en.wikipedia.org/wiki/Hessian_matrix

${ }^{12} \mathrm{http} / / /$ en.wikipedia.org/wiki/Jacobian_matrix_and_determinant
}

$$
R-R_{0}=\frac{\partial R}{\partial q}\left(q-q_{0}\right)
$$

In this process, $R$ matrix of radiative brightness temperature is square matrix. Therefore, it can be solved relatively easily.

The estimated matrix can be expressed as follows:

$$
\hat{x}=x_{a}+\left(A^{T} S_{\varepsilon}^{-1} A+S_{a}^{-1}\right)^{-1} A^{T} S_{\varepsilon}^{-1}\left(R-R_{a}\right)
$$

where $x_{a}, A, S, R_{a}$ denotes designated matrix, Jacobean matrix which is expressed with (12), measuring error covariance matrix which is expressed with (13), and designated radiative brightness temperature.

$$
\begin{aligned}
& A=\left(\begin{array}{ccc}
\partial R_{1} / \partial q_{1} & \partial R_{1} / \partial q_{2} & \partial R_{1} / \partial q_{n} \\
\partial R_{2} / \partial q_{1} & \partial R_{2} / \partial q_{2} & \partial R_{2} / \partial q_{n} \\
\partial R_{n} / \partial q_{1} & \partial R_{n} / \partial q_{2} & \partial R_{n} / \partial q_{n}
\end{array}\right) \\
& S_{i j}=\varepsilon\left(x_{i}-\hat{x}_{i}\right)\left(x_{j}-\hat{x}_{j}\right)^{T}
\end{aligned}
$$

Then, optimum solution can be obtained by Steepest Descent: Method: SDM method as follows:

$$
q_{k}=q_{k-1}+\alpha_{k} g_{k}
$$

where $\alpha, g$ denotes step size and direction of the next solution, respectively. Process flow of the SDM method can be represented in Fig. 3.

Initial value is very important. If the initial value is close to the global optimum solution, SDM allows to reach the global optimum solution easily while the initial value is far from the global optimum solution and is close to one of a plenty of local minima, then SDM reaches a local minima easily. In the proposed method, the initial value is given by Lear Estimation Method: LEM. Generally, it is considered the LEM gives a solution which is situated at an appropriate solution space not far from the global optimum solution. Therefore, it is expected that the proposed method allows much appropriate solution rather than the SDM with arbitrary initial value not from the LEM in terms of residual error and computational resources.

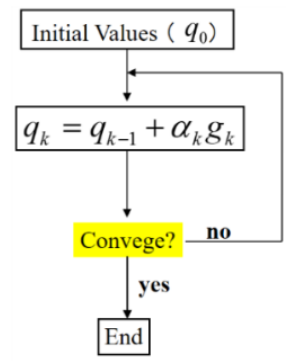

Fig. 3. Process flow of the SDM method. 


\section{EXPERIMENT}

\section{A. Preliminary Experiment}

AIRS data of the Mexican gulf at latitude of 20 degree North and longitude of 49-degree West which is acquired at 18:00 UTC on November 16, 2002 is used for the experiment. The intensive study area is shown in Fig. 4.

Also, Mid-Latitude Winter model of MODTRAN is used with the standard relative humidity, ozone and Carbone dioxide together with the standard water vapor and airtemperature profiles because the Mexican gulf is situated in the Mid-Latitude region.

From the AIRS data, 9 channels of data are selected as shown in Table I. The top of the atmosphere brightness temperature $(\mathrm{K})$ in the wave number range of $1460-1620 \mathrm{~cm}^{-1}$ is shown in Fig. 5.

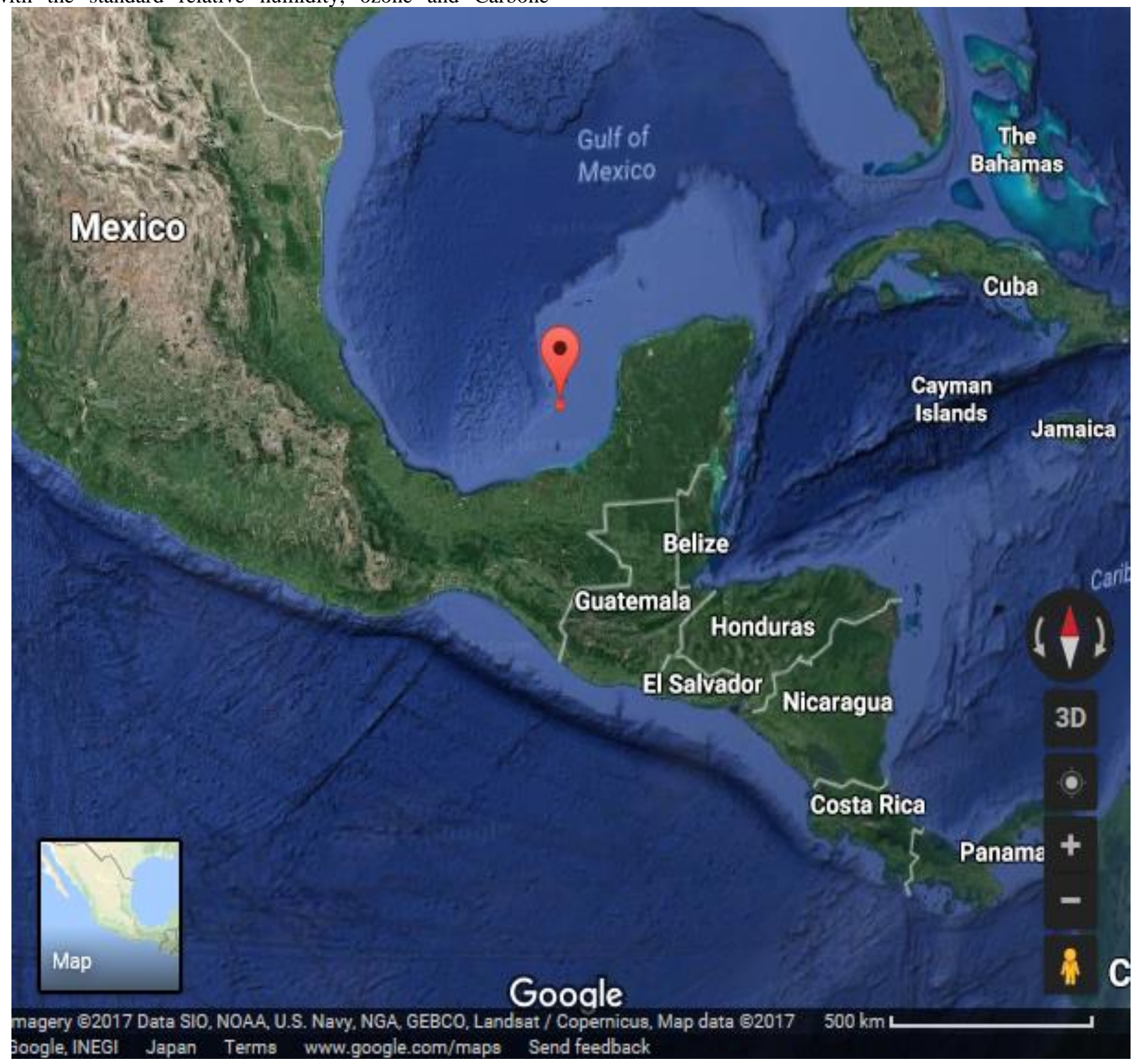

Fig. 4. Intensive study area.

TABLE I. SElEcted Airs ChanNels TOGETHER With Their WAVE Number

\begin{tabular}{|l|c|c|c|c|c|c|c|c|c|}
\hline WAVE NUMBER & 1478 & 1483 & 1508 & 1514 & 1519 & 1541 & 1544 & 1558 & 1585 \\
\hline AIRS CHANNEL & 1684 & 1692 & 1731 & 1740 & 1748 & 1761 & 1765 & 1786 & 1824 \\
\hline
\end{tabular}




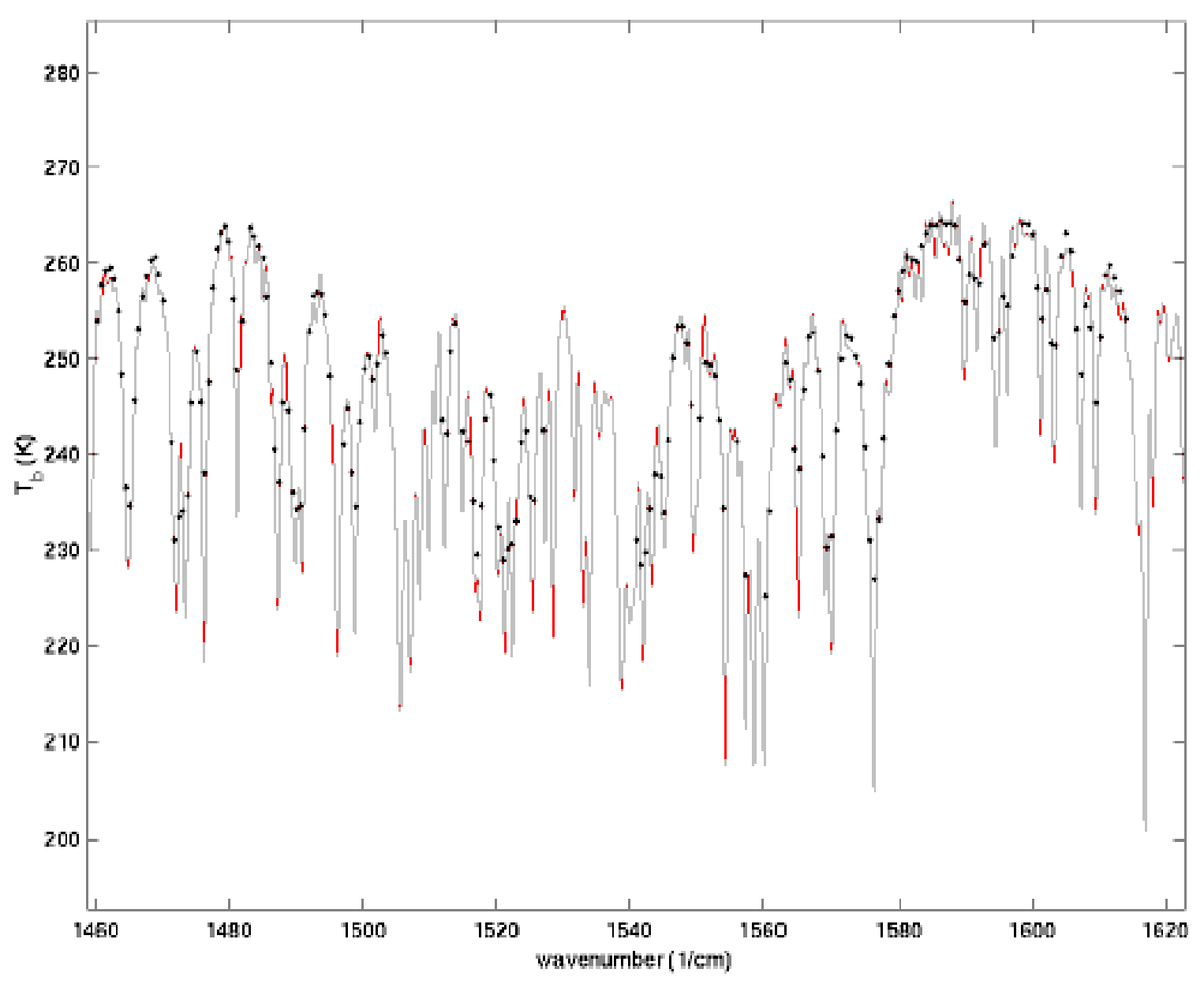

Fig. 5. The top of the atmosphere brightness temperature (K) in the wave number range of $1460-1620 \mathrm{~cm}^{-1}$.

The peaks of the weighting functions imply that the most sensitive altitudes to the relative humidity profile.

By using these brightness temperature and weighting functions, relative humidity profile can be estimated based on the proposed method with the SDM method. The procedure of the proposed relative humidity profile estimation is shown in Fig. 6. Meanwhile, weighting functions of these channels are shown in Fig. 7.

The most sensitive wave number of AIRS channels are selected. Then the top of the atmosphere brightness temperature is calculated with MODTRAN. Starting with the initial point $R H_{0}$ which is estimated by using the method based on linear model of problem solving, the best matched brightness temperature is to be found by changing relative humidity profile.

First, wave number together with the corresponding weighting function is selected. Then, the top of the atmosphere brightness temperature is calculated using MODTRAN. The best relative humidity profile is estimated by minimizing the difference between observed brightness temperature and the MODTRAN derived brightness temperature using the SDM method.

\section{Select Wave Number}

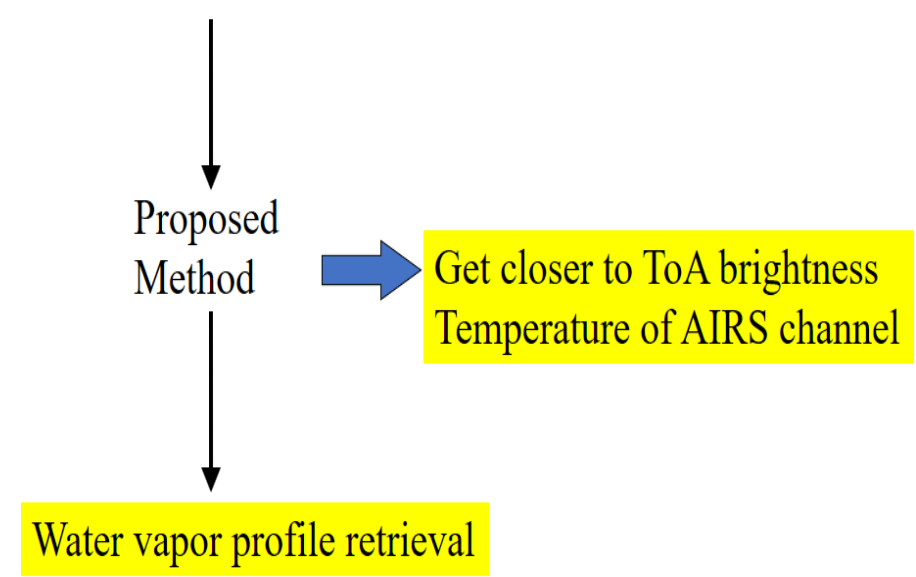

Fig. 6. Procedure of the proposed relative humidity profile estimation method. 


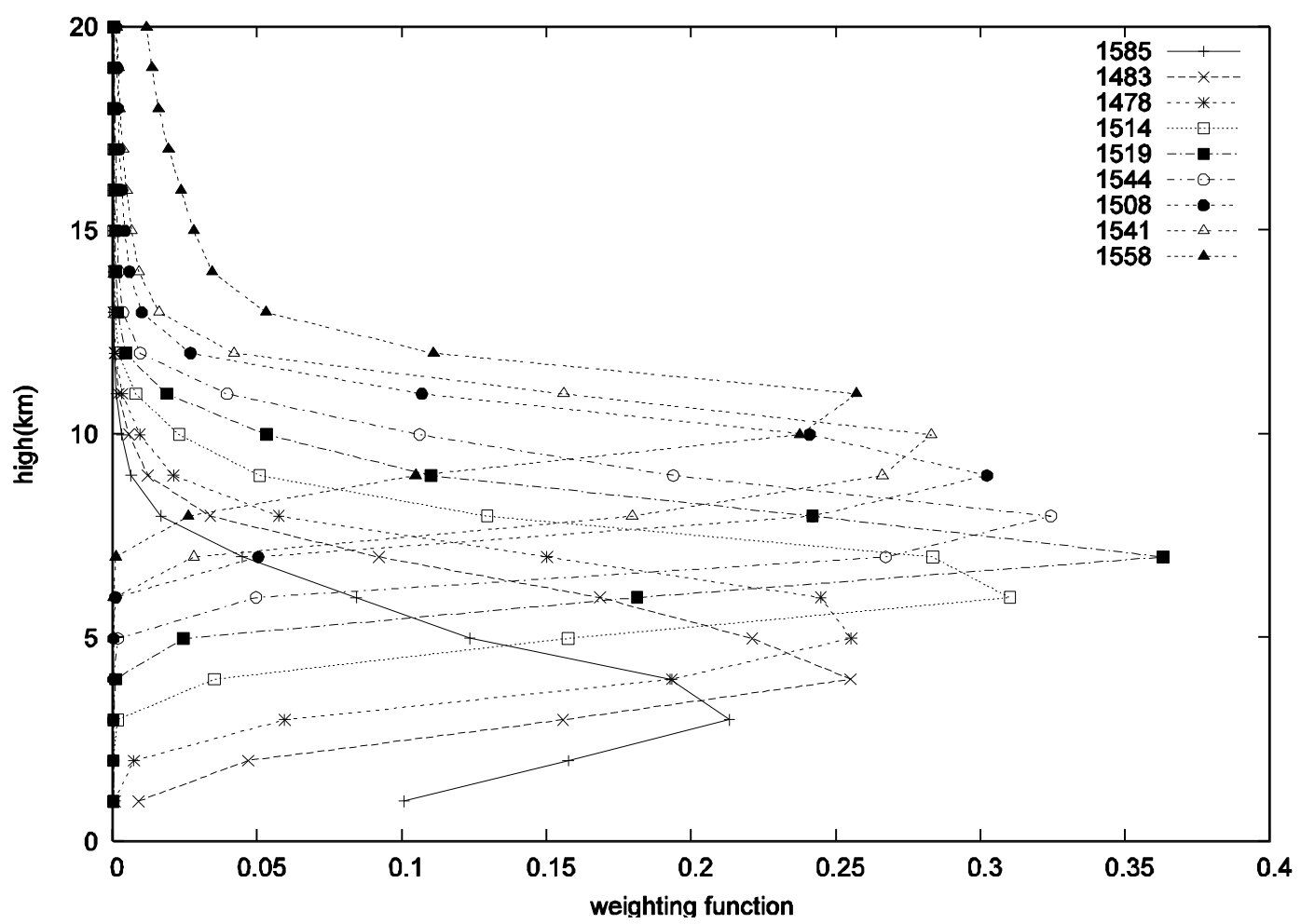

Fig. 7. Weighting functions of the selected 9 wave number channels

\section{B. Retrieved Result}

The retrieved result of relative humidity profile based on the proposed method is shown in Fig. 8. Meanwhile, the difference between calculated and acquired brightness temperature is shown in Fig. 9. There are three retrieved results in the Fig. 8, Mid-Latitude Winter, Mid-Latitude Summer and Tropic atmosphere models of MODTRAN. The AIRS data used is acquired in November 2002. Therefore, Mid-Latitude Winter would be better for relative humidity profile estimation. It, however, is not true in some cases as shown in Fig. 8. Trend of the retrieved relative humidity profile with Mid-Latitude Winter coincide to the MODTRAN derived profile.

Meanwhile, the difference between calculated and estimated brightness temperature shows different trends depending on the wave number. It is not always the difference decreases in accordance with increasing of altitude.

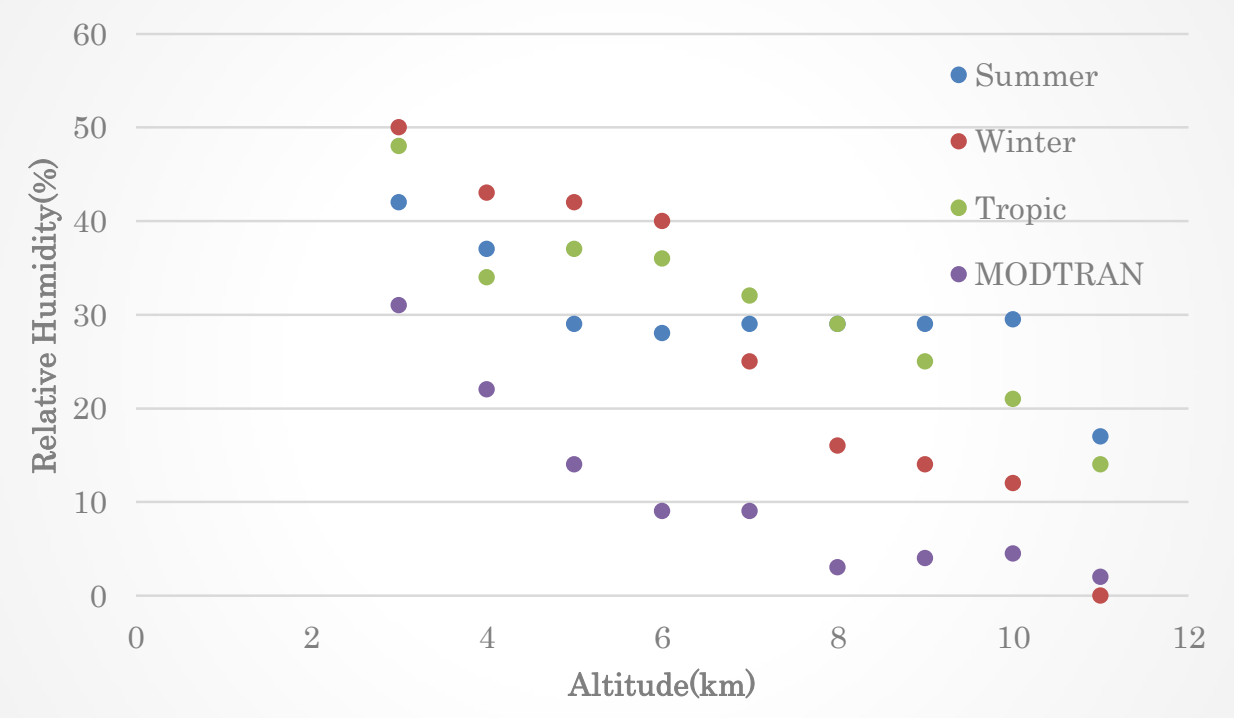

Fig. 8. Estimated relative humidity profile. 


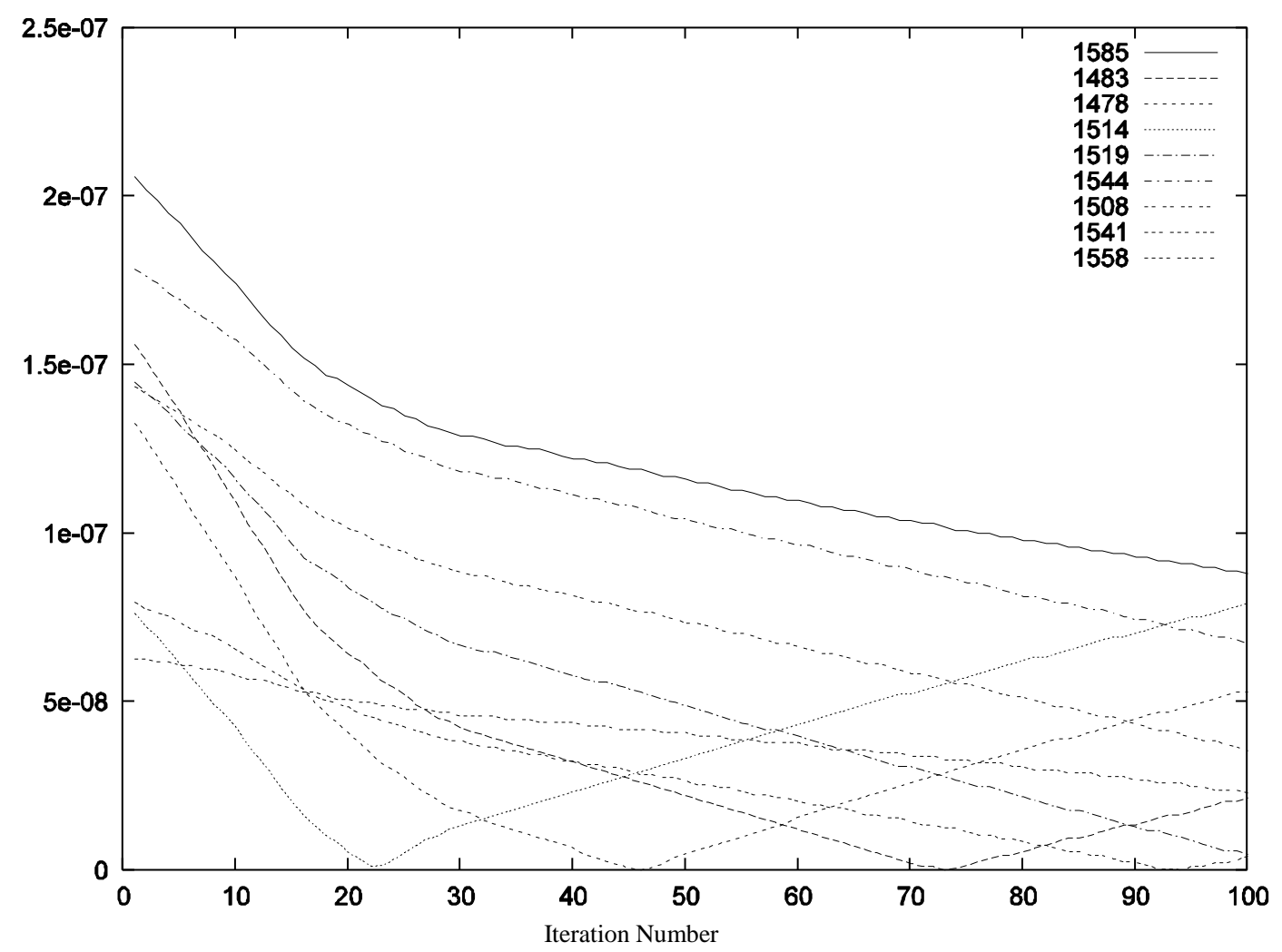

Fig. 9. Difference between calculated and acquired brightness temperature (K).

It is found that there is almost $15(\%)$ of relative humidity estimation error. Therefore, it can be said that the relative humidity is still tough issue for retrieval. It is also found that the estimation error does not depend on the designated atmospheric models, Mid-Latitude Summer/Winter, Tropic. Even if the assigned atmospheric model is not correct, the proposed SDM based method allows almost same estimated relative humidity. In other word, it is robust against atmospheric model.

\section{CONCLUSION}

Non-linear optimization method based on LevenbergMarquardt of non-linear least square method with numerically calculate deviation of Jacobean and Hessian is proposed for relative humidity and air-temperature profile estimations with infrared sounder data. Through comparisons with the conventional Newton-Raphson method, it is confirmed that the proposed method is superior to the conventional NewtonRaphson method in terms of retrieving accuracy. Also, the proposed method does not need Jacobean and Hessian because first and second order derivatives are calculated numerically with MODTRAN code based radiative transfer model.

It is found that there is almost $15(\%)$ of relative humidity estimation error. Therefore, it can be said that the relative humidity is still tough issue for retrieval. It is also found that the estimation error does not depend on the designated atmospheric models, Mid-Latitude Summer/Winter, Tropic. Even if the assigned atmospheric model is not correct, the proposed SDM based method allows almost same estimated relative humidity. In other word, it is robust against atmospheric model.

Further investigation is required for improvement of relative humidity retrieval accuracy.

\section{ACKNOWLEDGMENT}

The author would like to thank Mr. Noriaki Yamada of Saga University for his effort to conduct the experiments.

\section{REFERENCES}

[1] Kohei Arai, Lecture Note on Remote Sensing, Morikita-Shuppan publishing Co. Ltd, 2004.

[2] NASA/JPL, "AIRS NASA/JPL. http://airs.jpl.nasa.gov/overview/overview/.

[3] NASA "Aqua and the A-Train". NASA. http://www.nasa.gov/mission_pages/aqua/.

[4] NASA/GSFC "NASA Goddard Earth Sciences Data and Information Services Center". NASA/GSFC. http://disc.gsfc.nasa.gov/AIRS/data_products.shtml.

[5] NASA/JPL "How AIRS Works". NASA/JPL. http://airs.jpl.nasa.gov/technology/how_AIRS_works.

[6] NASA/JPL "NASA/NOAA Announce Major Weather Forecasting Advancement". http://jpl.nasa.gov/news/news.cfm?release=2005-137.

[7] NASA/JPL "New NASA AIRS Data to Aid Weather, Climate Research". NASA/JPL. http://www.jpl.nasa.gov/news/features.cfm?feature=1424.

[8] Kohei Arai and Naohisa Nakamizo, Relative humidity and airtemperature profile estimation with AIRS data based on Levenberg Marquardt, Abstract of the 50th COSPAR(Committee on Space Research/ICSU) Congress, A 3.1-0086-08,995, Montreal, Canada, July, 2008 
[9] Kohei Arai and XingMing Liang, sensitivity analysis for air temperature profile estimation method around the tropopause using simulated AQUA/AIRS data, Advances in Space Research, 43, 3, 845-851, 2009.

\section{AUTHOR's PROFILE}

Kohei Arai, He received BS, MS and PhD degrees in 1972, 1974 and 1982, respectively. He was with The Institute for Industrial Science and Technology of the University of Tokyo from April 1974 to December 1978 also was with National Space Development Agency of Japan from January, 1979 to March, 1990. During from 1985 to 1987, he was with Canada Centre for Remote Sensing as a Post Doctoral Fellow of National Science and Engineering Research Council of Canada. He moved to Saga University as a
Professor in Department of Information Science on April 1990. He was a councilor for the Aeronautics and Space related to the Technology Committee of the Ministry of Science and Technology during from 1998 to 2000. He was a councilor of Saga University for 2002 and 2003. He also was an executive councilor for the Remote Sensing Society of Japan for 2003 to 2005 . He is an Adjunct Professor of University of Arizona, USA since 1998. He also is Vice Chairman of the Science Commission "A" of ICSU/COSPAR since 2008 then he is now award committee member of ICSU/COSPAR. He wrote 37 books and published 570 journal papers. He received 30 of awards including ICSU/COSPAR Vikram Sarabhai Medal in 2016, and Science award of Ministry of Mister of Education of Japan in 2015. He is now Editor-in-Chief of IJACSA and IJISA. http://teagis.ip.is.saga-u.ac.jp/index.html 